\title{
Highlighting the Relevance of Motivation and Job Satisfaction of Nurses in Ghanaian Hospitals
}

\author{
Charles Owusu-Aduomi Botchwey ${ }^{1 *}$ \\ ${ }^{1}$ Department of Health Administration and Education, Faculty of Science Education, University of Education, \\ Winneba, Kumasi, Ghana
}

*Corresponding Author: Charles Owusu-Aduomi Botchwey, Ph.D. Candidate, Assistant Lecturer, Department of Health Administration and Education, Faculty of Science Education, University of Education, Winneba, Kumasi, Ghana. Tel: +233-555228869, Email: chaboat08@yahoo.com

Received March 13, 2021; Accepted May 16, 2021; Online Published May 22, 2021

\begin{abstract}
Background: Provision of quality and accessible health services comes with its challenges. Therefore, the need to highlight how motivation and job satisfaction can impact nurses' performances at the Korle-Bu teaching hospital.

Objectives: The research aimed to practically highlight nurses' motivation and job satisfaction by identifying what constituted motivation packages of nurses and how the work they did could give them satisfaction at the Korle-Bu teaching hospital.

Methods: The research employed a qualitative method with a case study design. In-depth interviews and focus group discussions (data triangulation) were used for primary data collection. A total of 40 respondents, including ten key informants, were selected using the purposive and convenience sampling techniques.

Results: The research found that the recognition of needs of nurses, improved remunerations, enhanced conditions of service of the nurses', regular payment of nurses' salaries', the institution of study leave with pay policy, among others, were considered as factors that could contribute to nurses' motivation and job satisfaction. These findings are consistent with the findings of other studies. Again, the study found that professional allowances paid to nurses, reduced rent, and the like are current motivation packages directed at nurses at the Korle-Bu teaching hospital.

Conclusion: It is important to state that for motivation to accomplish its intended purpose, there is the need for the hospital to identify the individual needs to satisfy to enhance job satisfaction.

Keywords: Motivation, Job Satisfaction, Remuneration, Salaries and Fringe Benefits, Hospitals, Health Services
\end{abstract}

\section{Background}

The competitive nature of today's business environment requires organizations to implement policies aimed at motivating employees and enhance employee job satisfaction. A commitment to improving satisfaction with work is key for the survival of organizations since organizations can only achieve their objectives when workers work in a supportive internal environment and are properly motivated to do so. However, adopting and maintaining a culture that promotes the motivation of employees and improving worker satisfaction remains a challenge for many organizations. ${ }^{1}$

According to Ahmed et al, ${ }^{2}$ motivation can be defined as recognizing workers' individual needs and adopting smooth processes to meet these needs. Employers are expected to work with employees to understand their individual needs and align them with their organization's needs. Motivating employees is an effective way of improving job satisfaction and reducing demotivation. ${ }^{2}$ Also, motivation has been conceptualized as "an encouragement that can optimize the potential of employees to work well. They successfully achieve and realize the goals that have been determined. ${ }^{3}$

Armstrong, ${ }^{4}$ in his book entitled "Motivation Strategies Aim at Creating a Working Environment," defined motivational factors as those tactics and strategies adopted by management to optimize employees' performance at work and improve the overall productivity of an organization.

Several psychological factors are known to influence the motivation of workers in an organization. One such psychological phenomenon that plays a key role in the motivation of employees is their satisfaction with the job. ${ }^{5}$ Whether or not employees are satisfied with their work has been objectively viewed in recent times as an indicator for worker motivation in most professions. This application of job satisfaction as an indicator for worker motivation has also been made to the nursing profession, one of the most physically, mentally, and emotionally demanding professions. ${ }^{5}$ It could therefore be said that one way to improve the output of nurses is through the assessment of

Copyright (C) 2021 The Author(s). This is an open-access article distributed under the terms of the Creative Commons Attribution License (http:// creativecommons.org/licenses/by/4.0), which permits unrestricted use, distribution, and reproduction in any medium, provided the original work is properly cited. 
job satisfaction which will ultimately improve the quality of their work and get them to be more motivated to work towards achieving organizational goals.

Živanović et $\mathrm{al}^{5}$ in their study of Serbian nurses, found considerably high levels of job dissatisfaction among the nurses. Based on evidence from the literature that links job satisfaction with worker motivation, the researchers concluded that the nurses had very low motivation levels. Given the strong bond between nurses' job satisfaction and their motivation to work, all high-level hospitals need to put in place an effective human resource management culture for nurses. ${ }^{6}$ Globally, nurses represent an essential part of the health workforce responsible for delivering quality healthcare. Therefore, improving nurses' working conditions and applying evidence from scientific studies represent a worthy investment in health. The Healthcare workforce and especially nurses, is generally limited, so there is the need to motivate the current workforce to be productive.

Studies have shown that when nurses are motivated and satisfied with their work, they become more productive and perform better. ${ }^{7,8}$ Several factors such as appropriate remuneration; availability of opportunities for further education, training, and professional development; improving living and work conditions; emphasizing open recognition; enhancing benefits and allowances; implementing decentralized structures; improving interpersonal relationships, effective communication and feedback; enhancing job descriptions, clearly established requirements for promotion and career development; use of a reward system; and promoting effective supervision and management have all been found to promote nurses motivation. ${ }^{9}$ For example, in a study in the Czech Republic, nurses reported that salaries and the quality of care for patients were the essential factors of consideration at the workplace. ${ }^{8}$

Moreover, favorable working conditions at the workplace are associated with higher levels of job satisfaction among nurses. Given that nurses play an important role in the delivery of care, there is a need for more research into what motivates nurses and increases the satisfaction of nurses in Ghana to inform national and facility-level policies and practices that will ensure the optimum utilization of healthcare resources.

\section{Objectives}

The purpose of the study was to assess the relevance of motivation and job satisfaction of nurses in Ghanaian hospitals, using the Korle-Bu teaching hospital as a case study. Specifically, the study sought to $(a)$ identify factors that can bring about motivation and job satisfaction among nurses at the Korle-Bu teaching hospital and $(b)$ assess the prevailing motivational packages directed at ensuring job satisfaction for the nurses at the Korle-Bu teaching hospital.

\section{Methods}

The study employed a qualitative method with a case study design. Qualitative research encompasses gathering and evaluating non-numerical documents.

The qualitative method enabled the researcher to obtain the subjective views of the respondents on the topic "Highlighting the Relevance of Motivation and Job Satisfaction of Nurses in Ghanaian Hospitals." The essence of using the qualitative method was to understand the concepts, opinions, and experiences of the topic from my selected respondents. The qualitative method helped to obtain in-depth experiences into the problem under investigation and generate new study ideas. In terms of data analysis, since the method was a qualitative approach, the study used the descriptive analysis method to analyze the data collected through the interview and the focus group discussion guides.

The two main instruments for data gathering were the interview guide and the focus group discussion guide. The interview guide enabled the researcher to have oneon-one interview sessions with the respondents to obtain their subjective views on the topic. The interview lasted for a maximum period of 45 minutes for each respondent. Respondents were well-oriented about the topic and the nature of the research before the commencement of the interview sessions. With the focus group discussion, the respondents were grouped into five. Each group consisted of eight members who were given questions relating to the topic and were asked to brainstorm to generate responses to the questions.

The research employed both convenience and judgmental sampling techniques to determine the sampling size from the total population. The total population was the entire Korle-Bu teaching hospital community with several departments and a very high Outpatient Department (OPD) attendance. In determining the sample size, convenience and judgmental sampling techniques were used. The sample size was arrived at by selecting two personnel from each department who were knowledgeable in nurses' motivation and job satisfaction. The judgmental sampling technique was applied to select the respondents who might matter in the topic under investigation.

The research method provided inclusion and exclusion lists since not all the personnel or populations found in the community of the Korle-Bu teaching were included or needed for the research. In the inclusion list, nurses who were twelve months old or more than twelve months were included in the sample size. Again, Senior Nursing Officers have also been included in the sample size and nurses at the rank of Deputy Director of Nursing. In the exclusion list, nurses who were less than twelve months were excluded from the sample size since a number of them did not have in-depth knowledge about their conditions of services and other remuneration.

The main variables in the study were motivation and job satisfaction. Under motivation, the study looked at factors that could motivate nurses at the Korle-Bu teaching 
hospital, such as conditions of service, remuneration of nurses, and other work-related conditions. The second variable was job satisfaction, and under that, the research looked at on-the-job factors that could provide job satisfaction for nurses at the Korle-Bu teaching hospital. Such factors might include the nature of the nurse's job, job enrichment, job enlargement, and simplification of nurses' jobs, among others.

The study used both primary and secondary sources of data. In this research, primary data were collected through a well-structured flexible interview guide and focus group discussions of five respondents in each group of eight to collect data on the following specific objectives: (1) Identify factors that can bring about motivation and job satisfaction among nurses at the Korle-Bu teaching hospital. (2) Assess the prevailing motivational packages directed at ensuring job satisfaction for the nurses at the Korle-Bu teaching hospital.

The researcher employed stenography, Grammarly, context, field notebook, and audio recorder in this qualitative study. The stenography assisted the researcher in recording respondents' responses in the shorthands to enable the interview process to move faster than expected without necessarily writing long sentences. The grammarly helped in identifying spelling mistakes and equally correcting grammatical errors during the report writing. The quetext helped to determine plagiarised contents and worked towards their rectification. Additionally, all ethical considerations were highly followed.

In using the in-depth flexible interview guide, questions were based on the two specific objectives. Permission was sought from respondents to record the interview between the researcher and the respondents, and where respondents declined to be recorded, field notes were taken. To solidify my responses from the respondents, a focus group discussion method was added to the interview guide, resulting in data triangulation.

The study also used the internet, journals, articles, reports, and other already published documents constituting the secondary data sources. The study population was the entire Korle-Bu teaching hospital community, and the sample size was forty. The study employed convenience and purposive sampling techniques to select knowledgeable respondents about the topic under investigation. In terms of data analysis, the study employed a descriptive analysis method. In selecting keywords for the abstract, the Mesh on Demand Browser was used to identify the keywords.

\section{Results}

To examine nurses' perception of motivation and job satisfaction in Ghana, nurses and management members were asked questions about; (1) factors that can bring about motivation and job satisfaction among nurses at the Korle-Bu teaching hospital. (2) the prevailing motivational packages directed at ensuring job satisfaction for the nurses at the Korle-Bu teaching hospital. The findings are presented in Table 1.

\subsection{Factors That Can Bring About Motivation and Job Satisfaction Among Nurses at the Korle-Bu Teaching Hospital}

The preliminary study revealed that several factors had contributed to nurses' motivation and job satisfaction at the Korle- $\mathrm{Bu}$ teaching hospital. The respondents explained that some of these factors were directly related to the various functions performed by nurses, while others were indirectly linked to the functions they performed as nurses. The study further found the nurses' motivation and job satisfaction at the Korle-Bu teaching hospital and the Ghana Health Service. In general, it could not solely be linked to either the nurses' desire to be associated with the nursing profession or the tangible benefits such as money, gifts, among others but other issues such as social recognition.

A nurse in the OPD explained that despite intermittent agitations of salary increments and better work conditions, if the activities and services of healthcare professionals, especially nurses at the Korle-Bu teaching hospital, could be given the needed recognition, the quality of healthcare would be enhanced. She argued that giving paramount recognition to the needs of workers was a very important prerequisite of ensuring successful quality healthcare delivery at the Korle-Bu teaching hospital. She added that the crucial assessment of managerial success is its capability to produce standards satisfactory to reward the nuisances imposed upon resources contributed. She lamented that:

Table 1. Findings of the Study Vis-à-Vis the Objectives

\begin{tabular}{|c|c|}
\hline Objectives & Findings \\
\hline & Nurses are motivated by social recognition \\
\hline & $\begin{array}{l}\text { Increased in salary, wages, and conditions of service of the nurses at the Korle-Bu } \\
\text { teaching hospital had contributed immensely to motivation and job satisfaction }\end{array}$ \\
\hline & $\begin{array}{l}\text { Setting equal performance standards for the nurses at the hospital had contributed to } \\
\text { the current motivation and job satisfaction }\end{array}$ \\
\hline & $\begin{array}{l}\text { Staff training through study leave with pay for nursing staff who might want to go for } \\
\text { further studies had effectively contributed to nursing motivation and job satisfaction }\end{array}$ \\
\hline \multirow{2}{*}{$\begin{array}{l}\text { Assess the prevailing motivational packages } \\
\text { directed at ensuring job satisfaction for the } \\
\text { nurses at the Korle-Bu teaching hospital. }\end{array}$} & $\begin{array}{l}\text { Nurses enjoyed allowances paid to them by the management of the facility for } \\
\text { providing services beyond the regular number of hours they were supposed to work }\end{array}$ \\
\hline & Cash was given to nurses at the end of the year in the form of motivation \\
\hline
\end{tabular}


"Even though we work to earn our salaries, I feel that nurses equally believe in the fact that when the general public recognizes our effort, we will do much better in quality service delivery."

A key informant mentioned that an increase in salary, wages, and conditions of service of the nurses at the Korle-Bu teaching hospital had contributed immensely to motivation and job satisfaction, leading to enhanced quality of healthcare delivery. The respondent explained that regular payment of nurses' salaries and improved conditions of service had inspired nursing workers at the hospital to contribute largely to healthcare delivery. The respondent added that despite an increase in the nurses' salaries, those ready to further their education could do so under the study leave policy instituted by the Ministry of Health through the Ghana Health Service. However, the respondent was quick to mention that despite the improved conditions of service, there were still dishonest nurses who were not performing up to the normal standards. The respondent opined that:

"There are some of our colleagues who are not working hard as if somebody forced them into the profession; I think there are others outside who are yearning to get this opportunity, but they are not getting and others have got, but they are abusing such glorious opportunity."

A nurse at the OPD added that comparing the nurses' conditions to other workers might have also motivated the nurses and increased job satisfaction. The respondent explained that by comparing the conditions of service of nurses to others whose qualifications were even higher, the nurses were deemed to be better off, which had increased their levels of motivation and enhanced their job satisfaction.

A management member of the hospital revealed that setting equal performance standards for the nurses at the hospital had contributed to the nurses' current motivation and job satisfaction at the hospital. The member explained that the introduction of equal standards of performance for all nurses with effective supervision had contributed to the high nursing staff motivation and job satisfaction. The member insinuated that:

"Management and board decided to ensure that all nurses have the equal number of times to attend to patients in the form of duty schedule and so no nurse or groups of nurses will feel that they are working many more hours than others."

The respondent explicated that in situations where some nurses felt that there had more tedious work schedules than others, it might result in internal wrangling, adversely affecting quality healthcare delivery at the hospital.

The study also revealed that staff training through study leave with pay for nursing staff who might want to go for further studies had effectively contributed to nursing motivation and job satisfaction at the Korle$\mathrm{Bu}$ teaching hospital. It was also reported that the study leave policy instituted by the Ministry of Health through the Ghana Health Service for the benefit of nurses and other healthcare professionals had increased the morale of nurses at the Korle-Bu teaching hospital.

\subsection{The Prevailing Motivational Packages Directed at Ensuring Job Satisfaction for the Nurses at the Korle-Bu Teaching Hospital}

The preliminary study revealed that many motivational packages had been instituted by the management of the Korle-Bu teaching hospital to ensure adequate job satisfaction of nurses and other healthcare professionals. The study found that the institution of these packages aimed to create a conducive work environment for the nurses and ensure that these nurses did not engage in unwarranted strike actions and demonstrations that could adversely affect healthcare delivery and quality of healthcare by these trained nurses.

Also, the study revealed that nurses of the Korle-Bu teaching hospital enjoyed some motivational packages aimed at promoting job satisfaction and enhancing the quality of healthcare. The study identified that nurses enjoyed allowances paid to them by the facility's management for providing services beyond the regular number of hours they were supposed to work. The study identified that such allowances were paid to the nurses as part of their salaries.

A nurse mentioned that those living on the premises of the hospital pay little rent for their accommodation. The nurse explained that those nurses who lived on the hospital premises paid much less rent than their counterparts who lived outside the hospital premises. The nurse mentioned that the hospital's management had failed woefully to ensure that nurses and other healthcare professionals at the facility were provided with quality accommodation befitting their status. The nurse lamented that:

"One of the few motivation packages we enjoy as nurses are the few of us who were fortunate to have accommodation on the premises paying much less than those living the towns of the metropolis."

The study further identified that cash was given to nurses at the end of the year in motivation. They revealed that the cash package was equally shared among the nurses as a mechanism for recognizing their effort in providing healthcare to the clients and patients of the facility. The study explained that such motivation was usually tied to the performance of the nurses and had usually compelled them to render much better quality services in the ensuing years. There meant that the more they rendered quality services to their clients, the more money they were likely to receive.

\section{Discussion}

Diverse scholars approve that nurses' motivation in healthcare organizations is appropriately important since nurses form a very important link in the healthcare value chain. Consistent with Onuoha ${ }^{9}$ findings, the study found social recognition as a key factor that could motivate nurses and increase their satisfaction. Therefore, managers of healthcare organizations in today's ever-evolving 
work environment need to implement policies beyond the tangibles to build trust and improve performance. ${ }^{10}$ The study also found that increase in salary, wages, and conditions of service of the nurses, regular payment of nurses' salaries, and availability of support for further education were key factors that motivated nurses at the facility. These findings are consistent with the general theories of motivation, such as the Motivation-Hygiene theory. Several studies have used this theory to study what factors motivate nurses and have found evidence that is consistent with the results of this study. ${ }^{11-13}$

The study also revealed high perceived levels of motivation and job satisfaction among the nurses compared to other professions due to opportunities for further study and the support instituted by the Ministry of Health. This finding contradicts what was found among Rwandan nurses in a study at Butare University teaching hospital, Rwanda. ${ }^{14}$ In this study in Rwanda, it was found that nurses in the hospital had a poorly motivated level. According to the authors, the low levels of motivation among the nurses resulted from dissatisfaction with working conditions, salaries, lack of opportunities for professional development, lack of recognition, benefits, allowances, and work-related rewards.

The study also revealed that the facility's management had instituted some packages for motivating the nurses and enhancing their satisfaction with the job. These packages included: allowances for extra hours of work, rent support for some nurses, and end-of-year cash packages. Contrary to the premises of Herzberg's theory, this study found cash packages to be associated with job satisfaction, and a similar finding was found by Holmberg et al. ${ }^{15}$ Moreover, empowering nurses, salary and financial packages, and contingent rewards, among many others, have been found to influence nurses motivation. ${ }^{16}$ In light of this, hospital managers must pay attention to issues that concern nurses' salaries since cash packages have been associated with satisfaction.

\section{Conclusion}

Per the results of the study, it can be concluded that some of the factors that motivated nurses were directly related to various functions performed by the nurses, while others were indirectly linked to the functions they performed as nurses. Nurses' motivation and job satisfaction at the Korle-Bu teaching hospital in particular and the Ghana Health Service could be linked to the nurses' desire to be associated with the nursing profession or tangible benefits such as money and gifts.

In identifying factors that could bring about motivation and job satisfaction, the study identified increased salaries, wages, and service conditions as factors that could enhance nurses' motivation and job satisfaction at the Korle- $\mathrm{Bu}$ teaching hospital. The study also identified that setting equal performance standards for nurses could spark nurses' recent motivation and job satisfaction at the KorleBu teaching hospital and staff training through study leave.

\section{Research Highlights}

What Is Already Known?

The motivation-hygiene theory holds that social recognition motivates employees and enhances their satisfaction.

\section{What Does This Study Add?}

- Nurses were highly motivated and satisfied due to opportunities for further study and support from the Ministry of Health.

- Contrary to the claims of Herzberg's theory, cash packages were found to be associated with nurses' job satisfaction.

In assessing the prevailing motivation packages of nurses of the Korle-Bu teaching hospital, the study concludes that the hospital's management had instituted many motivation packages. The essence of it was to create a conducive work environment for the nurses. The motivation packages may also include overtime allowances paid to nurses and reduced rent rates for living on the hospital and cash package premises.

It can be summed that the motivation packages used have been estimated to be effective and have been able to augment the performances and satisfaction of the nurses for whom they were intended.

\section{Conflict of Interest Disclosures}

The author declare that there is no conflict of interests.

\section{Ethical Approval}

This study was approved by University of Cape Coast Ethics Committee. Permission was sought from respondents and other bodies who mattered in the study and all other ethical protocols were duly followed right from the start of the study to the data collection process.

\section{References}

1. Sohail A, Safdar R, Saleem S, Ansar S, Azeem M. Effect of work motivation and organizational commitment on job satisfaction: (a case of education industry in Pakistan). Glob J Manag Bus Res. 2014;14(6):40-46.

2. Ahmed SM, Elsayed LA, El-Nagger NS. Effect of motivation versus de-motivation on job satisfaction among the nurses working in Hera general hospital at Makkah Al-Mukramh. Life Sci J. 2013;10(2):450-457.

3. Rahmah V, Sholihah Q, Gazali A, Sucipto. Relationship of motivation, compensation, and work environment with job satisfaction of nurses (Maslow's theory of motivation). Management in Health. 2018;22(2):21-23.

4. Britt TW. The effects of identity-relevance and task difficulty on task motivation, stress, and performance. Motivation and emotion. 2005;29(3):189-202. doi: 10.1007/s11031-0059441-3

5. Živanović D, Javorac J, Svitlica BB, Stojkov S. Job satisfaction as an objective indicator of work motivation among intensive care nurses. Int J Health Serv Res Policy. 2019;4(2):84-94. doi:10.23884/ijhsrp.2019.4.2.02. 
6. Rajan AV, Soni KD, Roja VR, Kamath R. Association between work motivation and job satisfaction among nurses in the clinical settings in India. Indian J Public Health Res Dev. 2019;10(12):1940-1943. doi:10.37506/v10/i12/2019/ ijphrd/192154.

7. Toode K, Routasalo P, Suominen T. Work motivation of nurses: a literature review. Int J Nurs Stud. 2011;48(2):246257. doi:10.1016/j.ijnurstu.2010.09.013.

8. Gomes F, Proença T. Nurses' Motivation and Satisfaction at Work: An Exploratory Study at the Centro Hospitalar S. João. 1st ed. Universidade do Porto, Faculdade de Economia do Porto; 2015. FEP Econ Manag [Internet]. 2015; Available from: http://wps.fep.up.pt/wps/wp558.pdf.

9. Onuoha P, Stephen A, Bernard P, Corban A, Mahabir M, Israel-Richardson D. Factors that contribute to work motivation and job satisfaction among hospital nurses in Trinidad and Tobago. Int J Health Sci Res. 2017;7(1):208217.

10. Sekhar C, Patwardhan M, Singh RK. A literature review on motivation. Global Business Perspectives. 2013;1(4):471487. doi:10.1007/s40196-013-0028-1.

11. Tan TH, Waheed A. Herzberg's motivation-hygiene theory and job satisfaction in the Malaysian retail sector: the mediating effect of love of money. Asian Acad Manag J. 2011;16(1):73-94.

12. Onuoha P, Ramcharan M, London N, Ramlal I, Sheen-Daniel L, Ezenwaka C. Motivational factors and techniques at the workplace: perspective of nurses in two major hospitals in Trinidad. Asian J Sci Technol. 2015;6(5):1388-1392.

13. Tan TH, Waheed A. Herzberg's motivation-hygiene theory and job satisfaction in the Malaysian retail sector: The mediating effect of love of money. Asian Academy of Management Journal. 2011;16(1):73-94.

14. Kamanzi J, Nkosi Z. Motivation levels among nurses working at Butare University teaching hospital, Rwanda. Afr J Nurs Midwifery. 2011;13(2):119-131.

15. Holmberg C, Sobis I, Carlström E. Job satisfaction among Swedish mental health nursing staff: a cross-sectional survey. Int J Public Adm. 2016;39(6):429-436. doi:10.1080/ 01900692.2015.1018432.

16. Baljoon RA, Banjar HE, Banakhar MA. Nurses' work motivation and the factors affecting it: a scoping review. Int J Nurs Clin Pract. 2018;5:277. doi:10.15344/23944978/2018/277 